\title{
Multilevel Petri Nets for the Specification and Development of Workflow Automation Systems
}

\author{
Marina Flores-Badillo and Ernesto López-Mellado \\ CINVESTAV Unidad Guadalajara \\ México
}

\section{Introduction}

Since its first use in 1980, the term Workflow has been gaining a lot of importance, especially in the improvement of organizational performance in a broad variety of industries (Shi et al., 1998).

Nowadays, Workflow Management represents a critical issue for achieving enterprise competitiveness among organizations, where workflow (WF) is concerned with the automation of procedures where documents, information, or tasks are passed to the participants according to a defined set of rules to achieve, or contribute to, an overall business goal (WfMC, 1999; van der Aalst \& Hee, 2002; van der Aalst, 1998). Workflow management aims to improve process performance and helps to achieve business goals with high efficiency (Reijers \& van der Aalst, 2005).

According to the Workflow Management Coalition, a WF Management System (WFMS), is "a system that defines, creates, and manages the execution of workflows through the use of software, running on one or more WF engines, which is able to interpret the process definition, interact with WF participants and, where required, invoke the use of IT tools and applications" (WfMC, 1999).

In WFMS development lifecycle, modelling is the first and most important stage and it lacks a standardized theory that provides a theoretical background (van der Aalst, 1998); that is why there are several modelling approaches which have their strengths and weaknesses in different aspects (Lu \& Sadiq, 2007). However, Petri Nets (PN) are usually used for formal modelling of WFs due to its formal semantics, graphical description, and abundance of analysis techniques (van der Aalst, 1996).

Since Zisman (Zisman, 1977) used PN to model WF processes, PN has been widely used for specifying, analysing and verifying the properties of static workflow models (van der Aalst, 1998; van der Aalst \& Anyanwu, 1999; Adam et al., 1998; Li et al., 2004), and extended for improve the expressiveness (Eshuis \& Dehnert, 2003; van der Aalst \& Hofstede, 2005).

Although PN provides clear and unambiguous models, when the systems are large and complex the handling and building of ordinary PN models becomes a difficult task.

Also, complex WFMS need to integrate other technologies, such as agent technology, which provides flexible, distributed, and intelligent solutions for business process management. 
The notion of agent in (Yan et al., 2001) is used as "a computer system situated in some environment, which is capable of autonomous action in this environment in order to meet its design objectives" (different notions can be found in (Wooldridge, 2002; Nwana, 1996)). These works also highlight the benefits of applying agent technology to business process (BP) management; some of these benefits are: distributed system architecture, the inherent autonomy of software agents because agents can start a WF based on event trigger, the agent reactivity because it have the ability to generate alternative execution paths, etc. An intelligent agent is capable of autonomous operation and flexible behaviour in order to meet its design goals and also has the properties of reactivity, pro-activity, and social ability (Wooldridge, 2001).

In other works both concepts are integrated. In (Repetto et al., 2003), a methodology for the design of agent based WF was presented; it consisted in three steps. In the first step the authors model the $B P$ with UML Activity diagrams by identifying all the necessary resources and activities. In the second step, all the activities identifying roles in parallel paths are grouped. Finally, they define an agent for each group.

A collaborative approach for workflow systems is presented in (Savarimuthu \& Purvis, 2004) where agents collaborate by forming social network (societies), in (Savarimuthu et al., 2004) agents are embedded in a system that can monitor and control the overall functioning of a workflow process in an agent based WF system.

In (Wang et al., 2005), agent technology is used for the WF monitoring where various intelligent agents work together to perform flexible monitoring tasks in an autonomous and collaborative way.

This work presents a method for the specification and development of agent-based workflow processes controllers. The proposed two phase methodology consists of a) the modular modelling of the system (using a PN based formalism called n-LNS); and b) an implementation technique using the obtained models and the middleware JADE (Bellifemine et al., 2007) as a development tool, where the models allow the synthesis of agent-based software in which mobile agents guide the process through organizational units and execute different tasks.

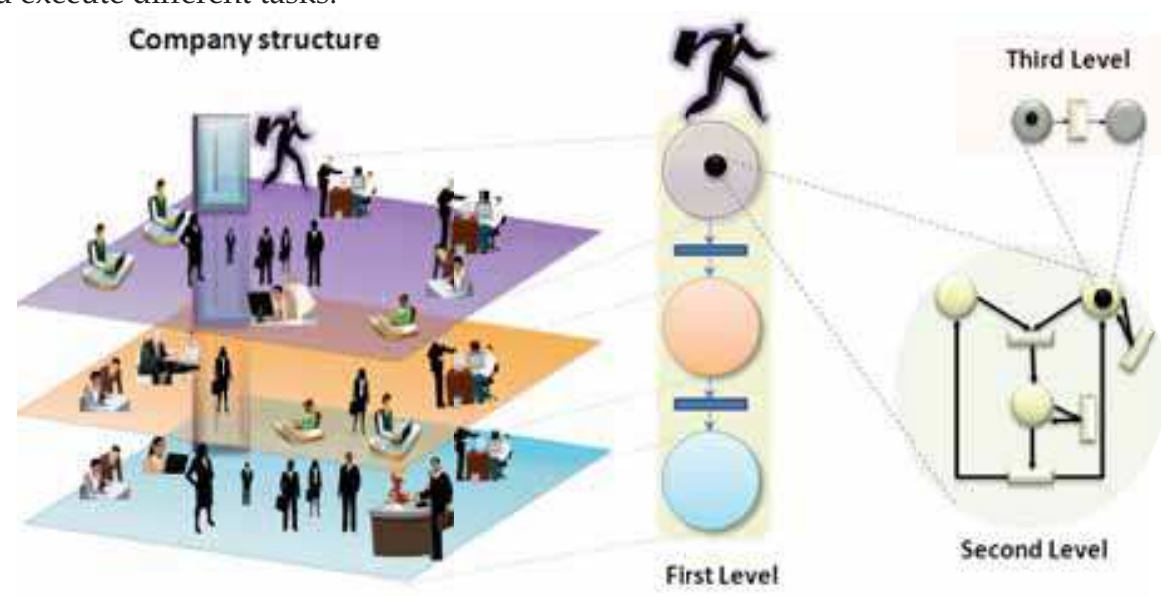

Fig. 1. General modelling strategy 
A workflow model may be clearly represented using three levels of the n-LNS formalism (see Fig. 1), where the first (highest) level describes the company structure and the possible flows between organisational units; the second level net describes the general behaviour of a entity which guides the processes within the company according to the defined workflow process definition, which is represented by a net of the third level; also the third level nets describe the tasks to be performed to complete the workflow case. The remainder of this chapter is organized as follows. Section II introduces the n-LNS formalism. In Section III we present a methodology for the modelling of workflows using n-LNS; simulation results are included. Section IV presents a software development technique using JAVA and the middleware JADE for component definition. Finally, Section V presents some current research and trends.

\section{A Multi-Level Net System}

\subsection{High level Petri Nets}

Among the existing modelling formalisms for discrete event systems, Petri nets (PN) have been widely adopted as a description formalism in the automation community, because of its characteristics issued of its graphical nature and its simple mathematical support: clearness and compactness to represent complex behaviour, namely causality, concurrence, parallelism, decisions, synchronization, and information exchange (DiCesare et al., 1993).

The largeness of PN describing complex systems motivated the creation of high level PN, extensions to the original formalism that allow compact models of systems. First, Predicatetransition nets proposed in (Genrich \& Lautenbach, 1979), and coloured PN (CPN), proposed in (Jensen, 1981), allow token identities (colours) represented by symbols in the marking. The expressiveness of CPN was increased by associating data structures to coloured tokens (Kasturia et al., 1988).

Later, high level modelling formalisms appeared to deal with object oriented software specification: concepts on high level PN and object oriented programming were merged leading to Cooperative Objects (Sibertin-Blanc, 1994) and Object Oriented PN (Lakos, 1995; Valk, 1991). In these works the tokens can be in turn other PN. These methods bring near the models to software implementation in despite of the loss of clearness of the description. Recently, the idea of considering PN as tokens is retrieved by R. Valk (Valk, 1998) who proposed a two-level PN “code-clean" formalism independent to programming languages. Holding the same notion of nets into nets, K. Hiraishi in (Hiraishi, 2000) proposes PN2, a two level formalism similar to the Valk's definition; also, I. Lomazova in (Lomazova, 2000) proposes Nested PN; also, in (Kummer, 2001) Reference nets are defined as a support of a simulation tool in which the tokens are references to other nets.

The proposed multi-level net system called $n$-LNS consists mainly of an arbitrary number of nets organized in $n$ levels according to a hierarchy; $n$ is chosen according to the degree of abstraction that is desired in the model. A net may handle similarly to tokens, nets of deeper levels and symbols; the nets of level n permits only symbols as tokens, similarly to CPN. Interactions among nets are declared through symbolic labelling of transitions. 


\section{$2.2 \mathrm{n}$-Level Net System}

The definition of $n$-LNS includes the description of the components (structure and marking), declaring of interaction (transition labelling), and the enabling and firing rules (embedding the synchronization mechanism). In this definition the basic notion of PN structure is used.

Definition 1. A PN structure is a triple $G=(P, T, F)$ where $P$ and $T$ are finite nonempty set of places and transitions respectively, $P \cap T=\emptyset$, and $F: P \times T \cup T \times P$ is a flow relation of the net. Pictorially, places are represented as circles and transitions as bars or boxes.

\section{- Type Nets}

Definition 2. A type-net of level $i$ is a tuple typenet $t_{i}=\left(G, T O K E N_{i}, L A B E L_{i}, V A R_{i}, \tau, \lambda, \pi\right)$ for $1 \leq i \leq n$, where:

- $\quad G$ is a PN structure.

- $\quad$ TOKEN $N_{i}$ is a finite non empty set of type-nets and symbols permitted into the places of a net level $i$ : TOKEN $_{i} \subseteq\left\{\right.$ typenet $\left._{i+j, k} \mid j=1, \ldots, n-i, k=1, \ldots, r\right\} \cup S Y M B O L_{i}$

$n$ is the number of levels of a multi level net system,

$r$ is the number of different type-nets allowed into places of a net of level $i$.

$S Y M B O L_{i}$ is a finite set of symbols allowed into the places of a net level $i$.

- $\quad L A B E L_{i}$ is a finite set of labels defined for a net level $i: L A B E L_{i} \subseteq L A B E L S$.

- $V A R_{i}=\{x, y, \ldots\}$ is a finite set of variables defined to net level $i ; V A R_{i} \subseteq V A R S$, where: Type: $V A R_{i} \rightarrow$ TOKEN $N_{i}$ is an assignment function of type nets to places.

$\operatorname{Type}(x)=\left\{\operatorname{Type}(x) \mid \forall x \in V A R_{i}\right\}$ is the set of types associated to variables.

- $\quad \tau: P_{i} \rightarrow 2^{\text {TOKEN }_{i}}-\emptyset$ is an assignment function of type nets to places.

- $\quad \lambda: T_{i} \rightarrow 2^{L A B_{i}}-\varnothing$ is an assignment function of labels to transitions where:

If $i=1$ then $L A B_{i}=L A B E L_{i}$

If $2 \leq i \leq n-1$ then $L A B_{i}=\left(L A B E L_{i} \cup\{\varepsilon\}\right) \times\left(L A B E L_{i} \cup\{\varepsilon\}\right) \times\left(L A B E L_{i} \cup\{\varepsilon\}\right)$

If $i=n$ then $L A B_{i}=\left(L A B E L_{i} \cup\{\varepsilon\}\right) \times\left(L A B E L_{i} \cup\{\varepsilon\}\right)$

- $\pi: F_{i} \times L A B E L_{i} \rightarrow M_{V A R_{i} \cup S Y M B O L_{i}}$ is a weighting function that assigns to every arc, a multi-set of variables and symbols, with respect to transition labels. If label $\notin$ $\lambda(t), \pi((p, t)$, label $)=\pi((t, p)$, label $)=\emptyset$. Moreover if $i=n$ then $V A R_{i}=\emptyset$, so that $\pi: F_{n} \times L_{A B E L_{n}} \rightarrow M_{S Y M B O L_{n}}$.

- $\quad$ LABELS and VARS are the sets of all the symbols representing the labels and variables respectively used in a model.

A type-net typenet $_{i}$ is a PN structure with additional information that declares and handles data defined in TOKEN $N_{i}$, according to the pre and post conditions established by $\pi$ and the labelling, specified by $\lambda$ for the interaction between nets.

The function $\tau$ assigns to every place a set of type-nets and symbols that belong to TOKEN . The function $\lambda$ assigns to every transition a set of labels. The labels of the lowest level are pairs: the first element indicates that the transition must be synchronized locally (with a transition of other net of the same level), and the second element indicates that the synchronization must be external (with a transition of a net of the next upper level), unless this pair is $(\varepsilon, \varepsilon)$. In the intermediate levels $(i=2, . ., n-1)$ a label is a triple: the elements in the triple declare local, internal (with a transition belonging to a token-net) and external synchronization, respectively only if the label is different form $(\varepsilon, \varepsilon, \varepsilon)$. The transitions of the highest level net are labelled either with a symbol or $\varepsilon$, declaring internal synchronization. The function $\pi$ determines on $(p, t)$ arcs, the amount and type of token-nets and symbols needed into the input places to enable a transition (those that must be removed), and on ( $t$, 
p) arcs, the amount and type of token-nets and symbols that must be added into the output places.

- Nets of level $i$

Definition 3. A net of level $i$ is a tuple $N E T_{i}=\left(\right.$ typenet $\left._{i}, \mu_{i}\right) ; 1 \leq i \leq n$, where:

- typenet $_{i}$ is a type-net of level i

- $\quad \mu_{i}: P_{i} \rightarrow M_{N E T S_{T O K E N}}$ USYMBOL $_{i}$ is a marking function for the type-net of level $\mathrm{i}$; it assigns to every place $P_{i}$ a multiset of nets and/or symbols.

$N E T S_{\text {TOKEN }_{i}} \subseteq\left\{N E T_{i+1}, N E T_{i+2}, \ldots, N E T_{n}\right\}$

A net of level $i$ is a type-net typenet $_{i}$ with a marking $\mu_{i}$, which provides the current distribution of symbols and nets of $\operatorname{NETS}_{\text {TOKEN }_{i}}$ into the places.

- Net System

A n-LNS model, called Net System, is the set of all nets defined at all levels.

Definition 4. A n-level Net System (n-LNS) is a n-tuple $N S=\left(N E T_{1}, N E T_{2}, \ldots, N E T_{n}\right)$ where:

- $\quad N E T_{1}$ is the highest level net

- $\quad N E T_{i}=\left\{N E T_{i, 1}, N E T_{i, 2}, \ldots, N E T_{i, r}\right\}$ is a set of $r$ nets of level $i$.

The Fig. 2 sketches pieces of the components of a 4-LNS. The level 1 is represented by the $N E T_{1}$, the level 2 by the nets $N E T_{2,1}$ and $N E T_{2,2}$, the nets $N E T_{3,1}, N E T_{3,2}, N E T_{3,3}, N E T_{3,4}$ and $N E T_{3,5}$ compose the level 3, and finally the nets $N E T_{4,1}, N E T_{4,2}, N E T_{4,3}$ and $N E T_{4,4}$ form the level 4.

\subsection{Net System Evolution}

The components of the model may interact among them through synchronization of transitions; the synchronization mechanism is included in the enabling and firing rules of the transitions. This mechanism establishes that two or more transitions labelled with the same symbol must be synchronized. In order to define the enabling conditions and firing of transitions we introduce first the notion of variable binding.

Definition 5. A binding $b$ on a variable set $\operatorname{VARS}=\{x, y, \ldots\}$ is a function $b: V A R S \rightarrow$ $\operatorname{NETS}_{\text {TOKEN }_{i}}$; for a $v \in V A R S ; b(v)$ is a next lower level net whose the type is Type $(v) . b_{t}$ maps every variable defined on the weight of the input arc to the transition $t$, with respect to a label. $m_{<b>}$ denotes a multiset of nets resulting of instancing a multiset of variables $m$ with the binding $b$.

- Enabling rule

Definition 6. A transition $t$ of a net of level $i N E T_{i}$ is enabled with respect to a label $l a b \in$ $\lambda(t)$ if:

- There exists a binding $b_{t}: V A R_{t} \rightarrow N E T S_{T_{O K E N} i^{\prime}}$ where $V A R_{t}$ is the set of variables appearing in all $\pi((p, t), l a b)$, and it must fulfill that $\forall p \in \bullet t, \pi((p, t), l a b)\left\langle b_{t}\right\rangle \subseteq \mu_{i}(p)$.

(The binding $\left\langle b_{t}\right\rangle$ is omitted when the level net is $n$, since the arc weight does not have variables. Therefore the condition is simplified).

- The conditions of one of the following cases are fulfilled:

Case 1. If $l a b=(\varepsilon, \varepsilon, \varepsilon)$ then no additional condition is required. The firing of $\mathrm{t}$ is autonomously performed.

Case 2. If $l a b \neq(\varepsilon, \varepsilon, \varepsilon)$ one must consider one or a combination of the following situations:

i) $l a b=(l, \varepsilon, \varepsilon)$. It is required the simultaneous enabling of the transitions labelled with $l^{\equiv}$ belonging to other nets into the same place $p^{\prime}$ of the next upper level net, marking 
the input places of $t$. The firing of these transitions is simultaneously performed and all the (locally) synchronized nets remain into the same place $p^{\prime}$.

ii) $l a b=(\varepsilon, l, \varepsilon)$. It is also required the enabling of the transitions labelled with $l^{\uparrow}$ belonging to other next lower level nets into $\bullet t$. These transitions fire simultaneously and the lower level nets and symbols declared by $\pi((p, t), l a b)\left\langle b_{t}\right\rangle$ are removed.

iii) $l a b=(\varepsilon, \varepsilon, l)$. It is also required the enabling of at least one of the $t^{\prime} \in p^{\prime} \bullet$, labelled with $l^{\downarrow}$, of the upper level net where the $N E T_{i}$ is contained. The firing of $t$ provokes the transfer of $N E T_{i}$ and symbols declared into $\pi\left(\left(p^{\prime}, t^{\prime}\right), l a b\right)\left\langle b_{t}\right\rangle$.

A label may involve a combination of any of these clauses. So, a label $(l, l, l)$ indicates that a transition must be synchronized locally, internally, and externally respect to the symbol $l \equiv \uparrow \downarrow$.

These situations apply to all levels of net, except for the nets of levels 1 and $n$. In the case of a net level 1 it is only possible the internal synchronization, so the transitions may be labelled with $l^{\downarrow}$ or $\varepsilon$. In the case of a net of level $n$ local and/or external synchronization may be declared; the transitions may be labelled with $l^{\equiv}, l^{\uparrow}, l^{\equiv \uparrow}$, or $\varepsilon$.

\section{- Firing rule}

The firing of transitions in all level nets modifies the marking by removing $\pi((p, t), l a b)\left\langle b_{t}\right\rangle$ in all the input places and adding $\pi((t, p), l a b)\left\langle b_{t}\right\rangle$ to the output places. The binding $\left\langle b_{t}\right\rangle$ is not necessary for nets of level $n$.

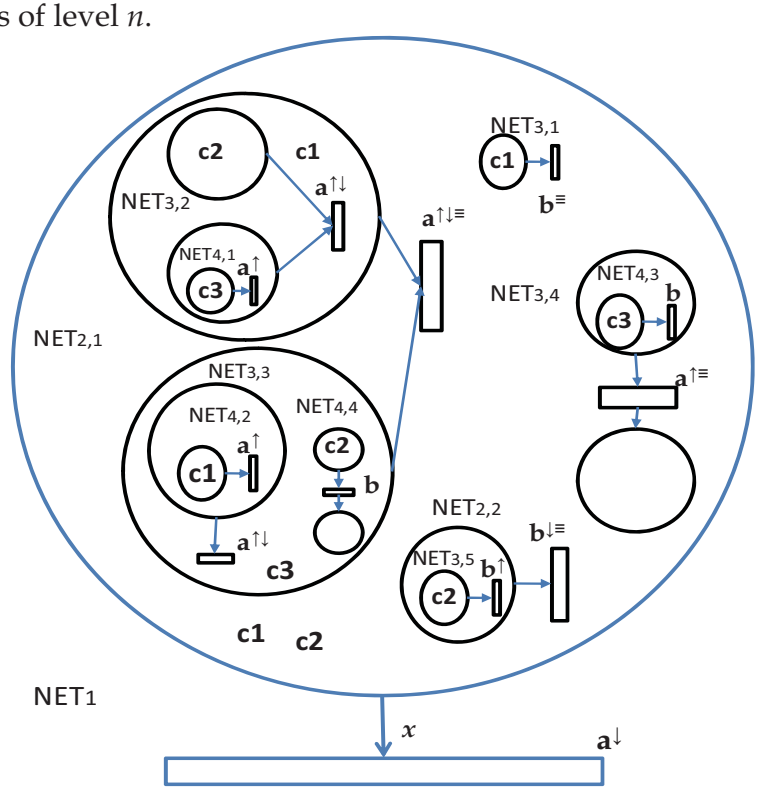

Fig. 2. Piece of a 4-Level Net System

In Fig. 2, $N E T_{1}$ is synchronized through the transition labelled with $a^{\downarrow}$ with $N E T_{2,1}$ and $N E T_{3,4}$ by mean of the transitions (locally synchronized) labelled with $a^{\uparrow}$; also $N E T_{2,1}$ is synchronized with nets $N E T_{3,2}, N E T_{3,3}, N E T_{4,1}$ and $N E T_{4,2}$ through transitions labelled with $a^{\uparrow \downarrow}$ and $a^{\uparrow}$; all these transitions must be enabled to fire. The simultaneous firing of the 
transitions removes these nets from the input places; $N E T_{4,1}$ is removed from the place of $N E T_{3,2}$, and $N E T_{4,2}$ is removed from the place of $N E T_{3,3}$.

$N E T_{2,2}, N E T_{3,1}$ and $N E T_{3,5}$ are synchronized through the transitions labelled with $b^{\downarrow}, b^{\equiv}$, and $b^{\uparrow}$ respectively; the firing of the transitions changes the marking of $N E T_{2,2}$ and $N E T_{3,1}$; $N E T_{3,5}$ is removed from the place of $N E T_{2,2}$.

\section{Workflow process specification}

\subsection{General Strategy}

The use of n-LNS induces a modular and hierarchical modelling methodology allowing describing separately the environment and the behaviour of all the involved components in a workflow problem, and then, integrated such models into a global one through transition synchronization.

As mentioned before, the workflow problem can be clearly described using only 3 levels of the n-LNS (see Fig. 1). The highest level net (1) describes the organization structure, i.e. the different organizational units involved in the solution of the workflow problem (case) and the relations (flow of information, tasks or documents) among them.

The second level net describes the general behaviour of an entity (software agent) which guides a process (current case) within the organizational units and executes the tasks within the departments expressed in the WF-process definition net (both types of nets modelled as nets of level 3 and described later); this entity net will be a token-net for the net of level 1 (Flores-Badillo et al., 2009a).

The method, for the sake of readability, is illustrated through a case study dealing with claim processes in an insurance company.

Consider the following description: "Define the WF for the claim processes in an insurance company in which a customer claims the insurance policy of a personal property (real state, car, life insurance). The company must receive the claim, request personal data from the customer (insurance policy number, etc.), and verify the insurance validity, payments, and beneficiaries. It must do the adjustment of real damages, validate the case, calculate the corresponding assessment, do the necessary payments to the customer if the complain is valid, or inform in case that the process has some invalid data".

The remainder of this section describes the definition of the different nets that compose the model at three levels.

\subsection{Level 1 Net}

This net describes the general structure of the company, where the WF processes are performed (environment net), and the possible workflow between every pair of organizational units or departments, considering all the possible cases. First, the different departments in which some tasks are executed are identified; then one must consider all the possible flows. The departments are represented by PN places and the flows by PN transitions (see Fig. 3), arc orientation corresponds to the direction of the flow. Firing of a transition means that the next task will be performed in the organizational unit that represents the output place of the fired transition. Furthermore, places for representing resources may be added in a classical structure allocation-release. 


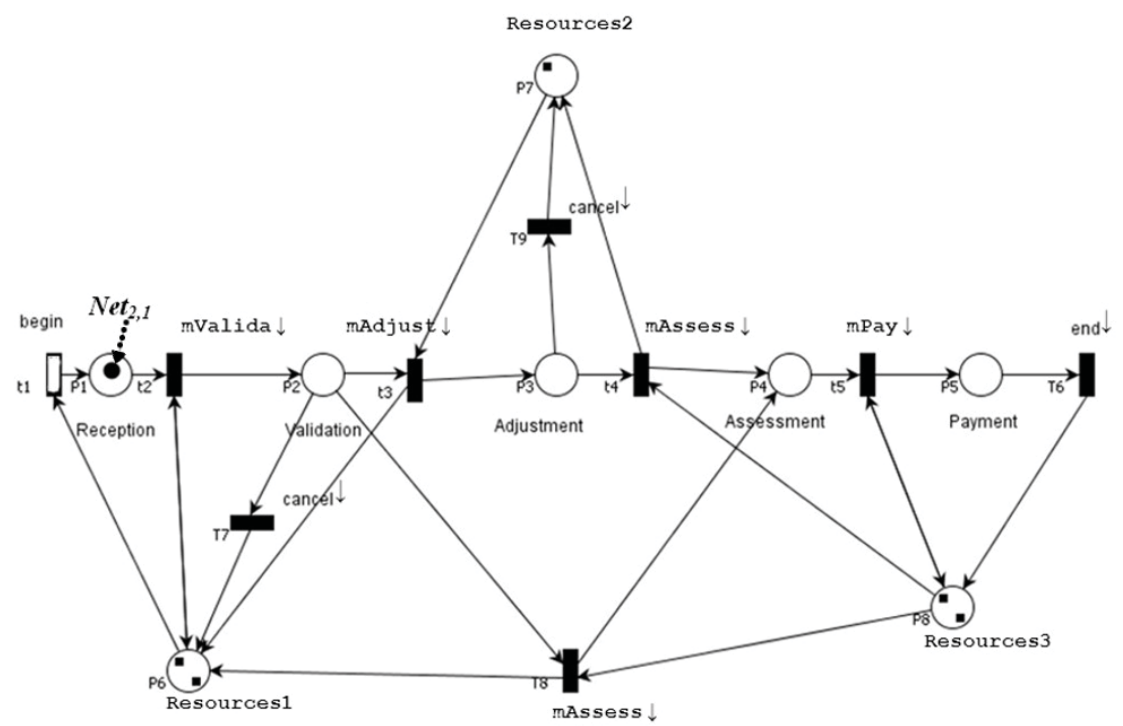

Fig. 3. Net ${ }_{1,1}$ net describing the organizational structure

Tokens in this net are nested nets of level 2; for example in place $P 1$ of the model of Fig. 3, there is a token $N e t_{2,1}$ which will be detailed later. If needed, transitions for allowing the cancelation of cases (see $t 7$ and $t 9$ in Fig. 3) can be added. Once this net is defined, synchronization attributes to the transitions must be declared. Also it is recommended using at least two transitions for representing the beginning and ending of a case. All transitions should include the internal $(\downarrow)$ synchronization attributes; it implies that the token-nets of level 2 must have some transitions labelled using the same symbols, with the external $(\uparrow)$ synchronization attributes.

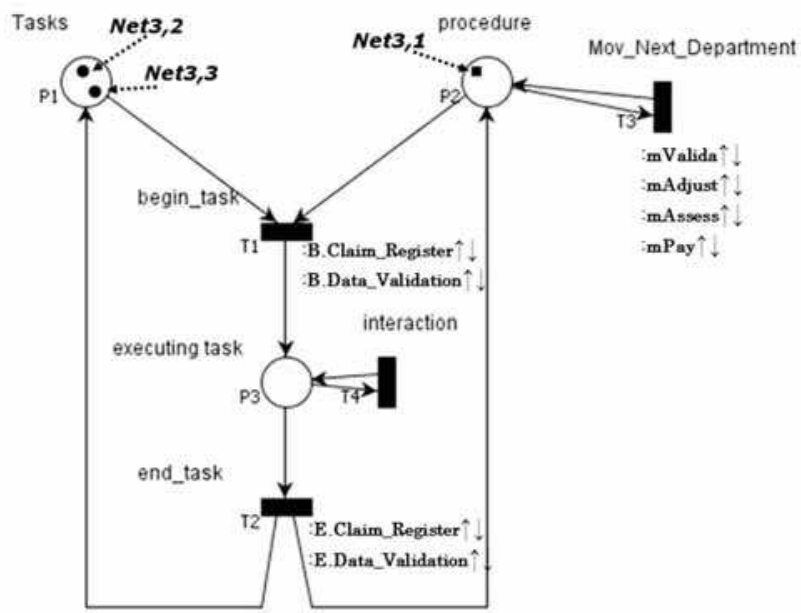

Fig. 4. Net 2,1 , net corresponding to a Mobile entity which handles the current WF case. 
The case begins with the firing of the transition $t 1$, and ends with the firing of the transition $t 6$ according to Fig. 3. Finally define $\operatorname{Net}_{1,1}=\left(G, \operatorname{TOKEN}_{1,1}, L A B E L_{1,1}, V A R_{1,1}, \tau, \lambda, \pi\right)$. We can also include in this model another kind of information, for example, the available resources for each department. This can be achieved adding places to the obtained net.

\subsection{Level 2 Net}

Define $\operatorname{Net}_{2,1}=\left(G, \operatorname{TOKEN}_{2,1}, L A B E L_{2,1}, V A R_{2,1}, \tau, \lambda, \pi\right)$, which models the general behaviour of an entity (mobile agent) that handles the case. This agent should know which tasks are involved in the WF process, in which department these tasks will be performed, and the pertinent information of the current case (that modify the order of tasks execution). Fig. 4 shows a simple agent net that handles as tokens, level 3 nets describing tasks and the WF process plan.

Initially, task nets $\left(\mathrm{Net}_{3,2}, \mathrm{Net}_{3,3}\right)$ reside in place P1, and the WF-process definition net $\left(\mathrm{Net}_{3,1}\right)$ is nested in place $P 2$ as initial marking. Net $_{3,1}$ determines which task (from all the available tasks in P1) has to be performed in the next step; when $T 1$ fires, both nets are placed into place P3 where the task nets evolves performing the involved transactions. When task is finished, this net enables the transition synchronized with $\mathrm{T} 2$ of $\mathrm{Net}_{2,1}$; the firing of T2 returns back the task net into $P 1$ and $N e t_{3,1}$ into P2. Both transitions (T1 and T2) must be declared with internal synchronization $(\downarrow)$. When the next task needs to be performed in a different department than the current, transition T3 must fire externally $(\uparrow)$ synchronized with the environment net $\left(\right.$ Net $\left._{1,1}\right)$ controlling the agent migration, and internally $(\downarrow)$ synchronized with $\mathrm{Net}_{3,1}$ because it defines the behaviour of the agent, i.e. the operations that it will perform. An additional transition (T4) can be included for interaction with other stationary or mobile agent nets.

\subsection{Level 3 Nets}

The nets of this level may be evolving within the agent net for providing a specific behaviour, namely the process plan, the tasks, or the agent interaction protocols. These nets may be within the environment model for specifying the state of resources or other stationary entities. For the case of study described, two types of level three nets are used: 1) those for describing the different tasks involved in the process of the case, and, 2) the net which describes the execution order of the tasks and the migration of the agent through the company (workflow process).

\section{- Net N $_{3,1}$ WF-process definition net}

The WF-Process definition net $\left(\mathrm{Net}_{3,1}\right)$, is a net having a simple structure and it must represent the possible sequences of tasks for accomplishing the handled cases of a WF process; the sequence include tasks executions and displacements between departments. Every stage in the plan is specified as place representing the tasks or the displacement operations; two transitions (as the labelled with B.Claim_register $\uparrow$ in T0 and E.Claim_register $\uparrow$ in T1 of Fig. 5) represent the beginning/ending of a task execution; each of this particular transitions must be synchronized with the corresponding net which models tasks and with the agent net Net $_{2,1}$.

The transitions representing displacements (as mValida $\uparrow$ ) must be synchronized with T3 of $\mathrm{Net}_{2,1}$. All the transitions must be externally $\uparrow$ synchronized. Finally define $\operatorname{Net}_{3,1}=(\mathrm{G}$, TOKEN $\left._{3,1}, \mathrm{LABEL}_{3,1}, \mathrm{VAR}_{3,1}, \tau, \lambda, \pi\right)$. An example of this net is showed in Fig. 5. 
- Net $t_{3, i}$ tasks nets

Each task is expressed as a simple sequence of transactions, where two transitions are used for emphasizes the beginning and the ending of a task execution. We can obtain each net identifying the operations required for each task and its precedence order. Pertinent labels are assigned for external synchronization with the agent net $\mathrm{Net}_{2,1}$, and local synchronization with Net $t_{3,1}$. For each task $i$ define the nets $\operatorname{Net}_{3, i}=\left(G, \operatorname{TOKEN}_{3, i}\right.$, $\left.\mathrm{LABEL}_{3, \mathrm{i}}, \mathrm{VAR}_{3, \mathrm{i}}, \tau, \lambda, \pi\right)$, for $i=2,3, \ldots . r, r$ is the number of tasks plus 1 . Fig. 6 shows the models of two tasks.

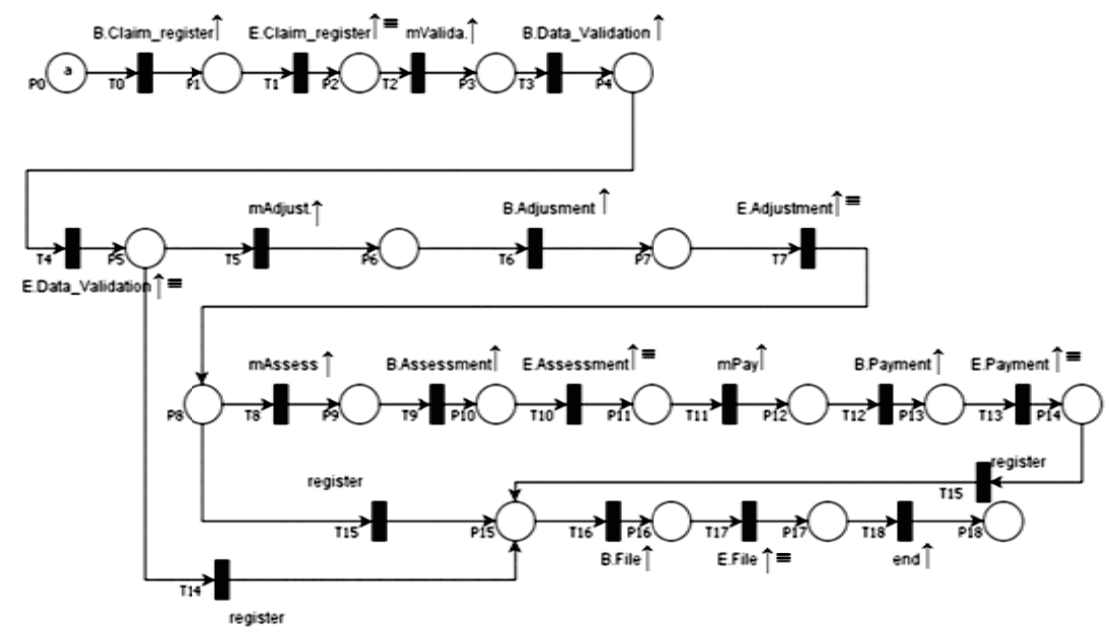

Fig. 5. Net ${ }_{3,1}$ defining a simple WF-process

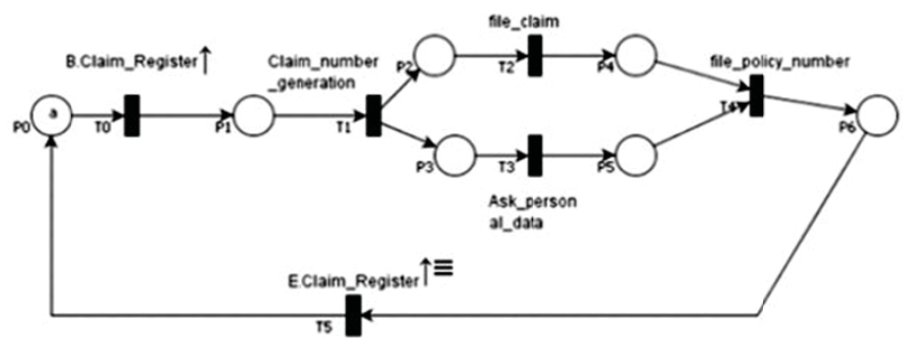

a)

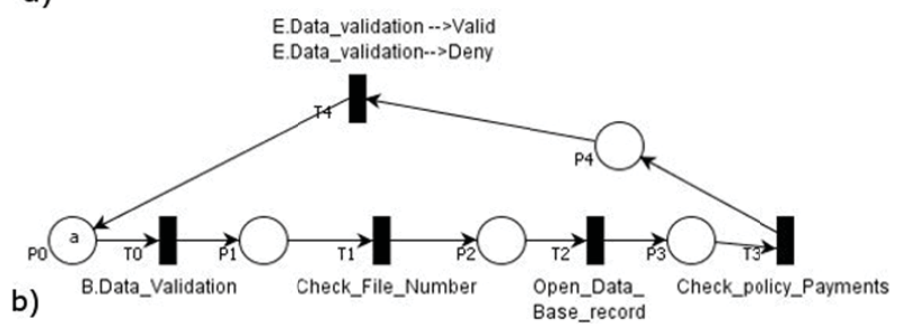

Fig. 6. Level 3 nets modelling two tasks: a) Net 3,2 for a Claim_Register task, b) Net $_{3,3}$ for a Data_Validation task. 


\subsection{Model Simulation}

Although the model construction is easily performed by following the proposed modelling methodology, the size of models becomes large as the complexity and quantity of activities in the system grow. In the absence of analytical procedures for verifying the correct functioning of the resulting models, interactive simulation appears as a useful and suitable solution for validating the obtained models. This task is enlightened by the use of a simulation software tool. In this section we describe the simulation procedure for n-LNS models and the tool for editing and executing such models.

\section{- Model Execution}

The simulation of n-LNS models consist in the interactive execution of the models according to the rules for enabling and firing transitions. We are going to briefly describe a procedure, usually named token player, which implements such rules.

The n-LNS models, edited through a visual interface, are coded into a XML file according to the standard format Petri Net Markup Language (PNML) (Weber \& Kindler, 2003); such file is called Data model. Besides the PN structure, other information is stored in the Data model, namely the level of the net, transition labels and their attributes, weight functions, marking, and Java code associated to transitions for software generation purposes.

From the Data model, a set of objects representing every $\mathrm{Net}_{i, j}$ are built. Every object evolves as the corresponding Net $_{i, j}$ by verifying the transition enabling conditions and the transition firing mechanism. Both enabling and firing procedures involve interactions among $\mathrm{Net}_{\mathrm{i}, \mathrm{j}}$ objects for checking synchronizations declared on the labels.

\section{- n-LNS Simulation Tool}

The simulation of the case of study has been performed through the execution of the 3-level net model described above. This task was possible with the help of MASGAS, a software tool that allows the visual edition and the interactive execution of multi level net models expressed in n-LNS.

The tool provides facilities for the interactive execution of the model: for a current marking the system indicates, through the highlighting of transitions, which transitions are enabled with respect to which label; then the user selects the transition to fire. After the firing the new markings of the involved nets are reached and displayed on the corresponding windows. Based on n-LNS, MASGAS provides a complete syntactic control over the different hierarchical levels of the net system; this avoids nesting of upper level nets allowed by other net within nets simulators (Kummer et al., 2002).

Below we are including several views of the edited model. Every net is built in a single window and it can be saved and updated for model adjustments.

In Fig. 7 it is showed the net which describes the company structure where the workflow tasks will be performed $\left(\mathrm{Net}_{1,1}\right)$, the token in $P 1$ is the nested net $\mathrm{Net}_{2,1}$ which models the general behaviour of the mobile agent that guides the handled case. Notice that the transition $t 2$ is enabled (respecting to the label $m$ Valida); when it is fired, the agent migrates from one organizational unit (Reception department in P1) to another (Validation department in P2). In Fig. 8 the $\mathrm{Net}_{2,1}$, is showed; the edited net shows the defined initial marking. At this situation the entity is ready to perform the initial task in the pertinent department. When transition $\mathrm{T1}$ of $\mathrm{Net}_{2,1}$ is fired, it means that the net contained in its place P2 (net Net se $_{3,1}$ in Fig. 5) indicates that a particular task (one task net from the available in P1) need to be performed and will be selected (through transitions synchronization). 


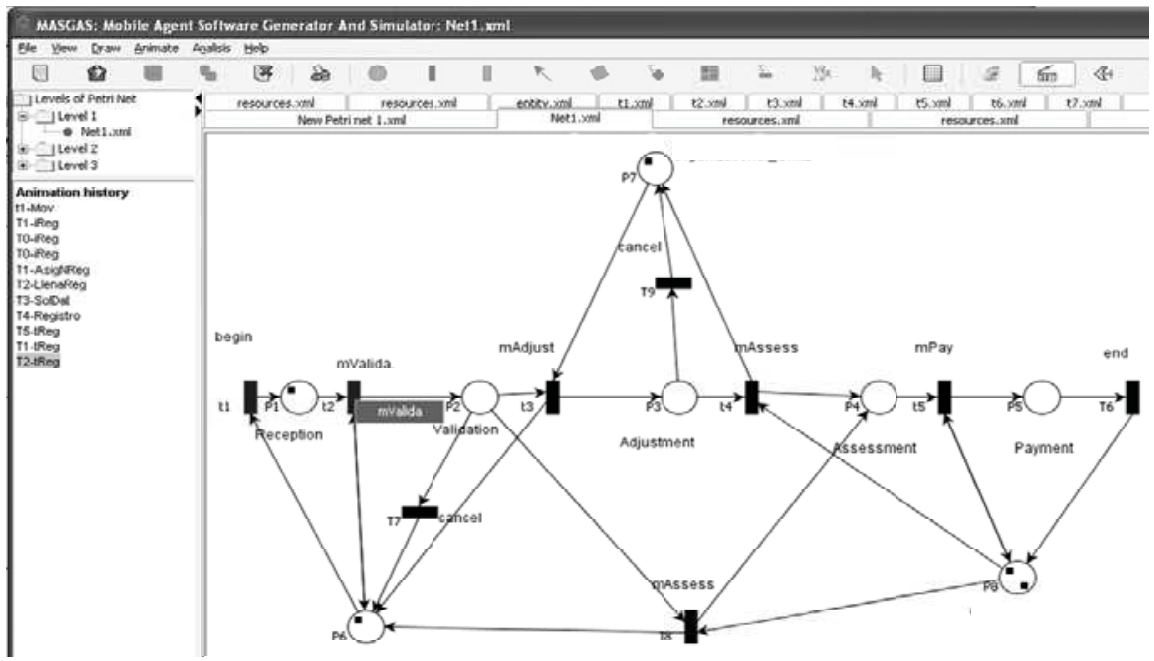

Fig. 7. Net $t_{1,1}$ (Organizational Unit Net): $\mathbf{t} 2$ is enabled with respect to $m$ Valida

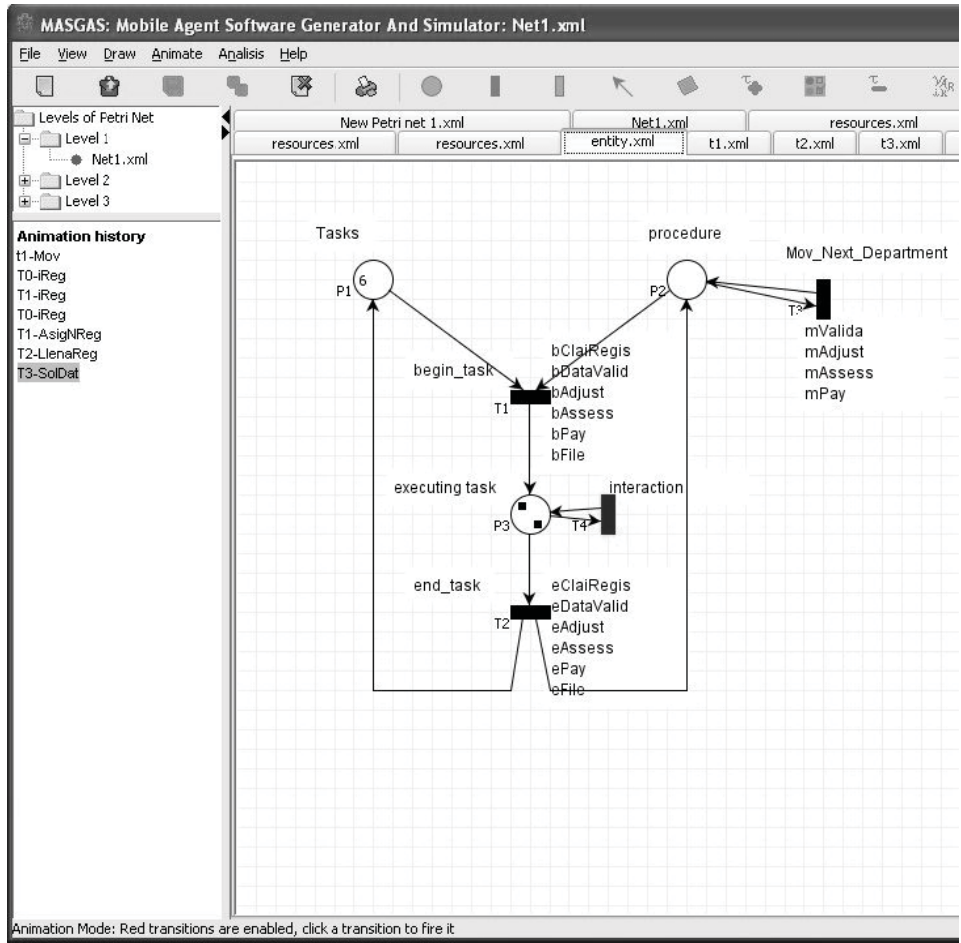

Fig. 8. Level 2 net (Mobile entity Net), after the firing of the transition T1: a token net of place $P 1$ and the token net of place $P 2$ are placed into $P 3$ 


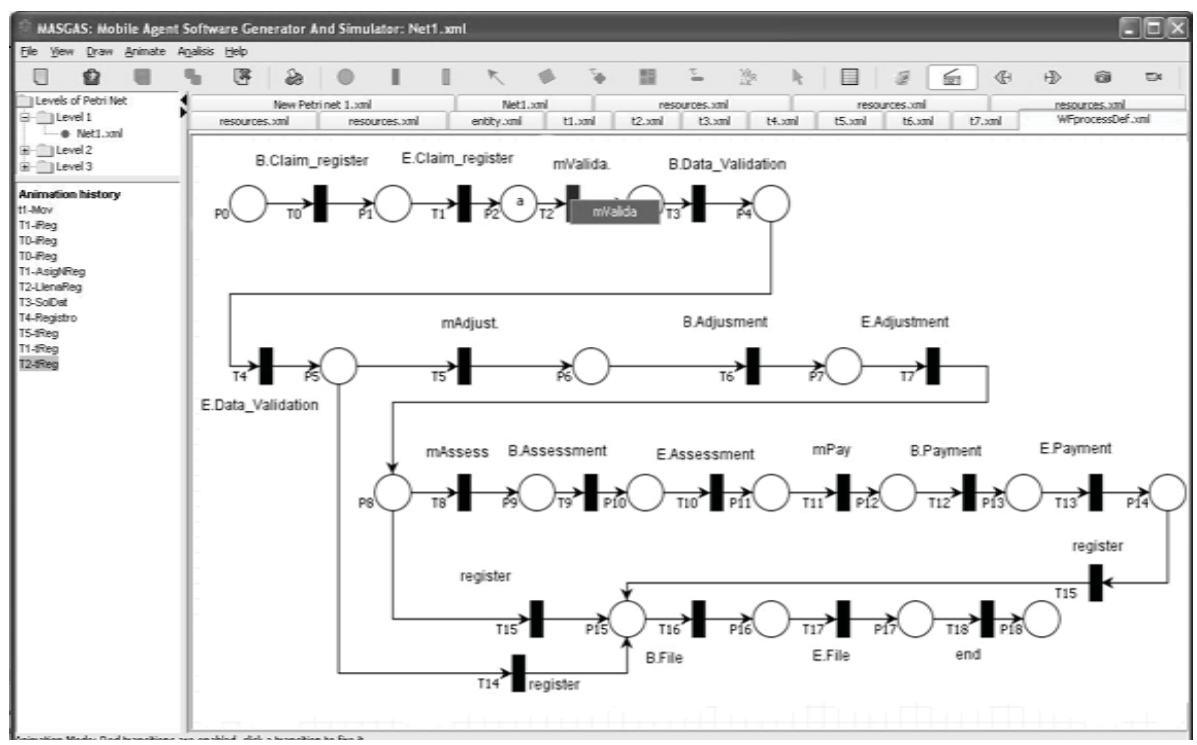

Fig. 9. Net 3,1 ; T2 (label $m$ Valida) is enabled: the entity need to migrate to the Validation Department (P2 in Net $\left._{1,1}\right)$.

Fig. 9 shows the edited model for the WF-process definition Net Net $_{3,1}$. When the given task needs to be performed in a different department than the current one, the transition Move_Next_Department of the Net $_{2,1}$ in Fig. 8, is used; it indicates that the entity migrated to other department within the organization (label mValida in Fig. 9).

\section{A Software Development Technique}

\subsection{Overview of JADE}

Java Agent Development Framework (JADE) is a software framework completely implemented in Java language which simplifies Mobile Agent (MA) system implementations by using a middleware which fulfill FIPA (FIPA, 1997) specifications. The agent platform can be distributed through machines (which not necessary share the same OS) and the configuration can be managed by a remote GUI (Bellifemine et al., 2007).

The communication architecture offers flexible and efficient message passing where JADE creates and manages the incoming private ACL message queue for each agent. The complete FIPA communication model has been implemented and its components have been clearly distinguished. JADE integrates completely interaction protocols, ACL, ontology's, transport protocols, etc. Most of the FIPA defined protocols are available in JADE.

\subsection{Software architecture}

This section presents a technique for the development of Mobile Agent-based WFMS. The basic idea for conceiving such a system is that a MA guides the workflow process through the different organizational units in which several tasks are executed according to the 
handled case. The application, obtained from an n-LNS model, runs concurrently in a set of networked computers where mobile agents execute concurrently the WF process for the different cases, interacting with stationary (software or human) agents.

During the design phase the components are described in a clear and compact way. The system is described as a set of interconnected organizational units that have a specific resource allocation. The agent behaviour is determined by two kinds of specifications: a) the description of the agent general behaviour and common knowledge for all the agents, namely, basic operations, and interaction protocols (collaboration, and resource competition); and b) particular descriptions of a specific behaviour such as the task plan and an accessibility roadmap, which describe the assigned process and the permitted access to the organizational units respectively.

The implementation phase is supported by a software development guideline allowing the definition of Java components (using also the middleware JADE) from agent systems specification from the design phase. The obtained software is distributed in a set of networked computers that manages MA migration. The modularity allows adaptations to system specification changes without difficulties.

Below an outline the main steps of the software synthesis methodology is presented, where the Mobile Agent Environment is first defined, and then both general and specific agent behaviours are described. All the system components are defined using the information given by the Nets obtained in the modelling phase.

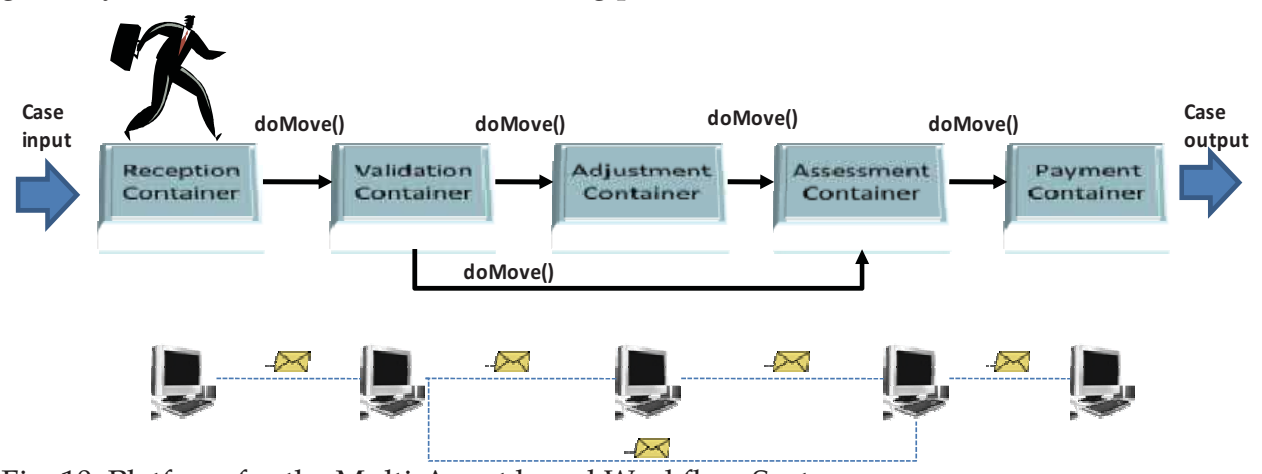

Fig. 10. Platform for the Multi-Agent based Workflow System

\section{- Environment Structure}

This is defined using the information given by the $\operatorname{Net}_{1,1}$, net which describes the general structure of the company; where each place of this net (representing a different department) can be seen as a site (host, container) of the Organization Local Area Network (LAN), placed each one in a different office; every site will contain an agent platform, so each transition of the Net $_{1,1}$ represents a migration of an agent from one site to another. In this way we can create a different JADE agent container for each place in that Net; a container can be created using: C: $\backslash$ java jade.Boot -container Name_Host [-gui]. In Fig. 10 a strategy for the platform distribution is proposed, but this can have a different distribution due to the company's net organization. 


\section{- Defining Agents with JADE}

The class for deriving all the mobile agents is defined extending the basic JADE class Agent. The common base class jade.core.Agent provides all the necessary features to accomplish basic interactions with the agent platform, where the setup() method registers an agent in the Directory Facilitator (DF) and deregister it with the takedown() method.

Furthermore, since mobile objects are used, one must register the SLCodec language and the MobilityOntology by adding the lines:

getContentManager().registerLanguage(newSLCodec());

getContentManager().registerOntology(MobilityOntology.getInstance());

which are also included in the setup() method (see Fig. 11).

In Fig. 11 an Agent general structure is described, where the specific behaviour of this agent must be added in the highlighted part of the code, defining all tasks that this agent should perform is order to process the WF case. This agent functionality is defined using the level three nets defined in the modelling phase.

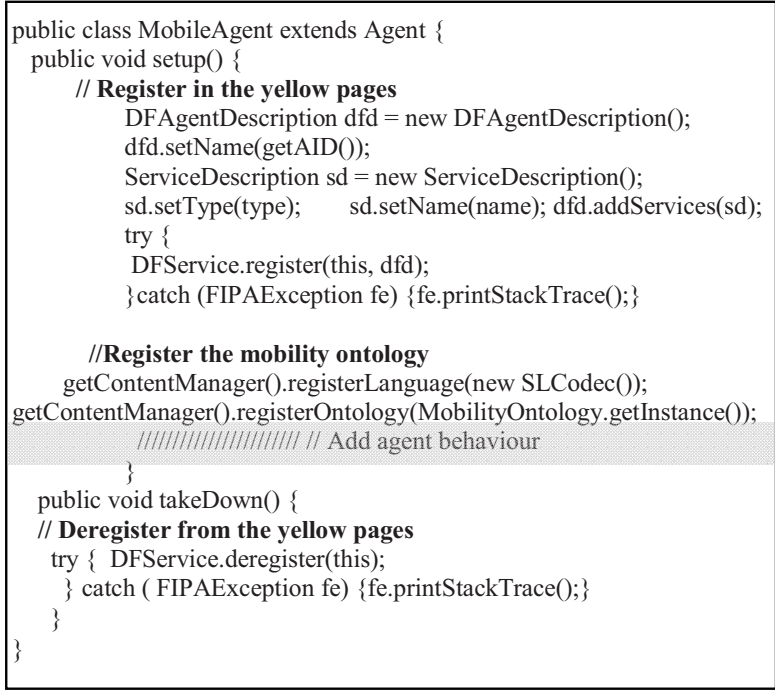

Fig. 11. Fragment of code for Agents Definition in JADE

\section{- Defining Agent functionality (agent behaviour) with JADE}

In JADE each functionality or service provided by an agent should be implemented as one or more behaviours that can be executed concurrently (Bellifemine et al., 2007). A behaviour is basically an event handler that describes how an agent reacts to an event; a behaviour is implemented as an object of a class that extends of: jade.core.behaviours.Behaviour, where one must to implement the action() method (the operations to be performed when the behaviour is executed) and the done() method (that specifies whether or not a behaviour has completed, through the Boolean value it returns).

The behaviours help to define the procedures specified by the level 3 nets in a n-LNS model. JADE provides ready to use complex behaviours that contain sub-behaviours and execute 
them according to some policy. Deciding about which behaviour to use depends of the specification given in the level 3 nets; however the structure of these nets usually is close to a Finite State Machine (FSM), and then the JADE's FSMBehaviour() can be used. In the used case of study, the Net 3,1 (modelled in Fig. 5) exhibits such a behaviour.

Other level 3 net structures may be closer to other behaviours, namely ParallelBehaviour(), SequentialBehaviour(), CompositeBehaviour(), and SimpleBehaviour().

Similarly, WF tasks can be programmed using the same behaviour. Fig. 12 shows the different methods used for defining the FSMBehaviour() corresponding to WF process net (Net 3,1 in Fig. 5); such methods declare (register) the transitions and the states of the equivalent FSM. Each state is registered using: registerFirstState(new Behaviour_Name(), State_Name) for the first state, and registerState(new Behaviour_Name(), State_Name) for the rest of them. Each transition is registered using: registerDefaultTransition(State1_Name, State2_Name), and registerTransition(State1_Name, State2_Name, value) used when a state have multiple outputs.

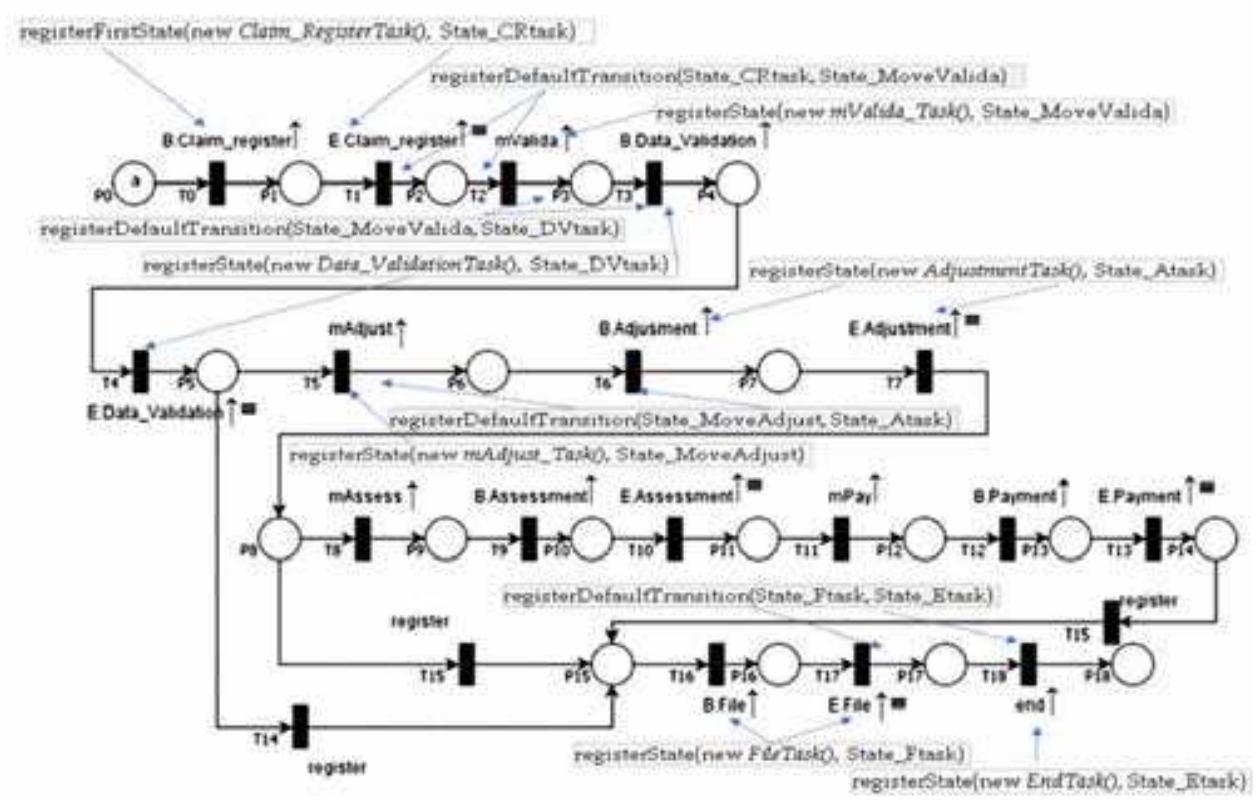

Fig. 12. Some states and transitions registrations for a JADE's FSMBehaviour()

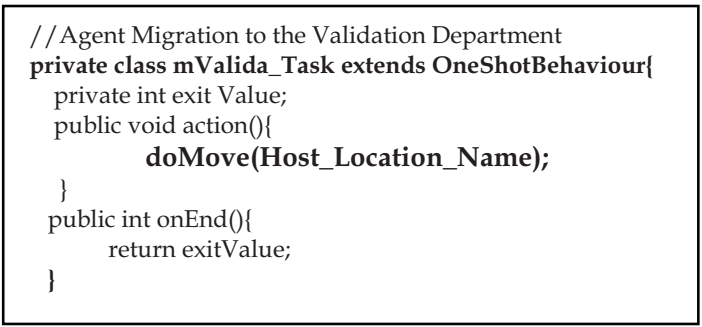


Fig. 13. Example of a Behaviour definition

In this way, each state of the FSMBehaviour() (for example state State_MoveValida from Fig. 12) will be a task or action that the agent will perform; the code for these tasks can be obtained from the nets modelling the involved WF tasks $\left(\mathrm{Net}_{3,2}, \mathrm{Net}_{3,3}, \ldots\right)$; it is placed in each Behaviour_Name() class added in the states registration. For example mValidaTask() behaviour registered in Fig. 12 represents the agent migration behaviour to the Validation Department; the appropriated code for the agent migration that must be added could be as showed in Fig. 13.

- Running the agent System with JADE

In Jade, for executing the agent it is necessary

a) compile it (javac-classpath <JADE-jars> AgentClassName.java), and

b) start it from JADE runtime environment (java -classpath <JADE-jars> jade.Boot Agent_Name:AgentClassName).

\section{Current research and trends}

The proposed methodology for modelling and developing workflow automation systems allows addressing the problem in complex organisations including several business partners (Inter-organizational WF). The technological constraints are limited to holding in the computer equipment the Java virtual machine and JADE supporting the containers that handle the agents.

However new problems appear when the partners share resources and WF processes, and the agents must migrate from one company network to another one (through internet) in order to process a handled case (see Fig. 14). Reliability and security issues must be taken into account during the design of the agent management to provide fault tolerance capabilities. Delays and lose of agents must be distinguished and opportune detected.

Current research addresses these issues as a problem agent population control (FloresBadillo et al., 2009b). A first protocol has been proposed including the tasks location, loss detection, and recovery of mobile agents; the protocol is supported by the handling of allowed timing in agent mission execution, allowing passive and active termination of agents, opportune localization of agents and orphan detection.

\section{Conclusions}

This work presented a method for developing workflow automation systems. A mobile agent based approach is proposed for obtaining distributed controller software. The first stage consists of a modelling methodology that uses n-LNS, yielding modular and hierarchical descriptions capturing both the agent environment and the agent behaviour. The programming stage allows creating straightforwardly from the obtained model, efficient and extensible software.

The proposed implementation technique uses JADE getting all the JAVA advantages. The mobile agent can interact with other agents for collaborate, negotiate or compete for resources.

Mobile agent based approach is suitable for distributed WF automation because it allows operating within an open platform architecture along local or remote locations. 


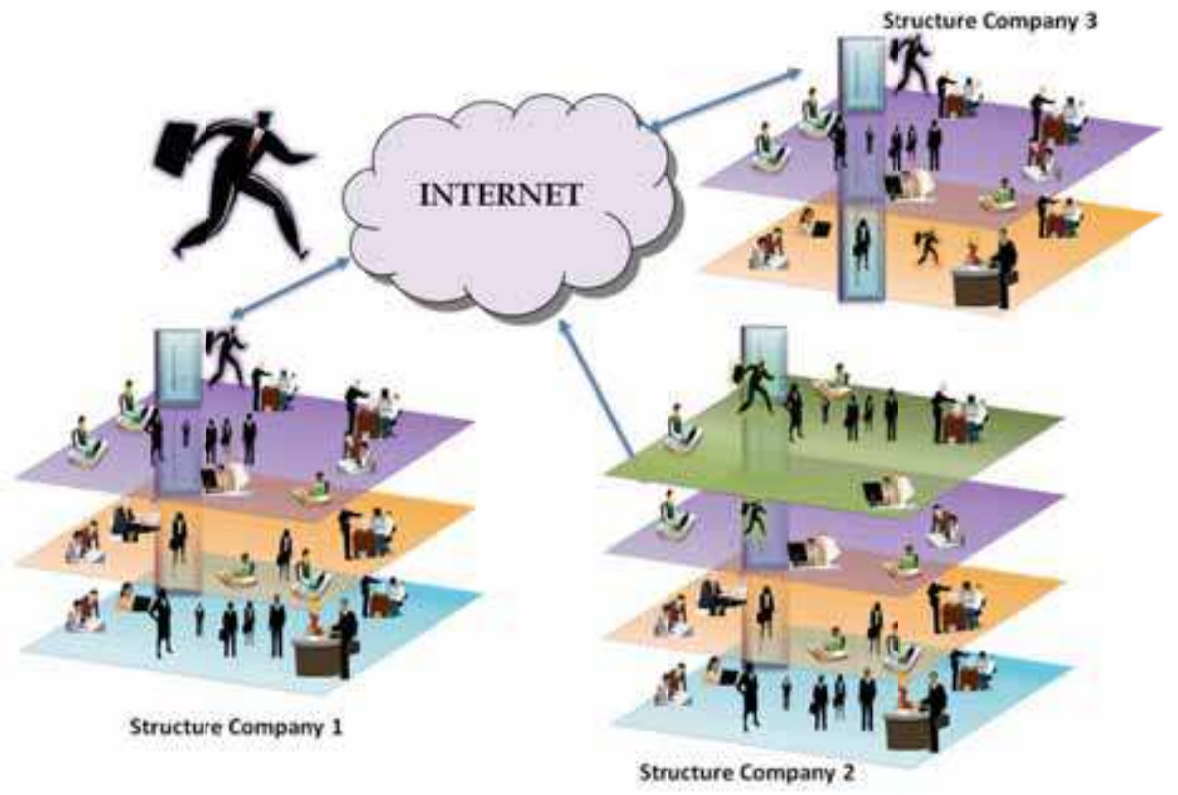

Fig. 14. Inter-Organizational Workflow System

\section{References}

Adam, N.R. ; Atluri, V. \& Huang, W.K. (1998). Modeling and Analysis of Workflows Using Petri Nets. Journal of Intelligent Information Systems. Vol. 10, Issue 2, March 1998, pp. 131-158, ISSN : 0925-9902.

Bellifemine, F.; Caire, G.; Trucco, T. \& Rimassa, G. (2007). Jade programmer's guide, JADE 3.5. 2007.

Dicesare, F.; Harhalakis, G.; Proth, J.M.; Silva, M. \& Vernadat, F.B. (1993). Practice of Petri Nets in Manufacturing, Chapman \& Hall.

Eshuis, R. \& Dehnert, J. (2003). Reactive Petri Nets for Workflow Modeling. In Application and Theory of Petri Nets 2003, vol. 2679, of Lecture Notes in Computer Science, pp. 296-315, ISBN: 3-540-40334-5, Springer Verlag, Berlin.

FIPA (1997). Foundation for Intelligent Physical Agents, Specifications. Available at: http://www.fipa.org.

Flores-Badillo, M.; López-Mellado, E. \& Padilla-Duarte, M.(2009a). Modeling and Simulation of Complex Workflow Processes using Multi-level Petri Nets, International Journal of Simulation and Process Modelling (IJSPM), Vol. 4, No.3/4, pp. 205-214, 2009. ISSN: 17402123.

Flores-Badillo, M.; Padilla-Duarte, A. \& López-Mellado, E. (2009b). A Population Control Protocol for Mobile Agent Based Workflow Automation, In: Proceedings of IEEE Int. Conf. on Systems, Man, and Cybernetics, pp. San Antonio, Texas, USA, October 2009. 
Genrich, H.J. \& Lautenbach, K. (1979). The Analysis of Distributed Systems by Means of Predicate/Transition-Nets, In: Semantics of Concurrent Computation, Lecture Notes in Computer Science, Vol. 70, Gilles Kahn (Ed), pp 123-146, Springer Verlag, ISBN : 9783-540-09511-8, Evian, France.

Hiraishi, K. (2000). A Petri-net-based model for the mathematical analysis of multi-agent systems, Proceedings of the IEEE International Conference on Systems, Man \& Cybernetics, Vol. 4, pp. 3009-3014, ISBN: 0-7803-6583-6, Nashville, Tennessee, USA, October 2000,

Jensen, K. (1981). Coloured Petri Nets and the Invariant Method, Theoretical Computer Science, Vol. 14. North-Holland, pp. 317-336.

Kasturia, E.; Dicesare, F. \& Desrochers, A. (1988). Real Time Control of Multilevel Manufacturing Systems using Colored Petri Nets, In: Proceedings of IEEE Int. Conf. on Robotics and Automation, Vol. 2, pp. 1114-1119, Philadelphia, Pennsylvania, USA, May 1988. Washington: IEEE Comput. Soc. Press.

Kummer, O. (2001). Introduction to Petri nets and Reference nets, Sozionik Aktuell, No. 1, pp. $1-9$, ISSN 1617-2477.

Kummer, O.; Wienberg, F. \& Duvigneau M. (2002) Renew - User Guide, University of Hamburg, Department for Informatics, Theoretical Foundations Group.

Lakos, C. (1995). From Coloured Petri Nets to Object Petri Nets, In Proceedings of $16^{\text {th }}$ International Conference on the Application and Theory of Petri Nets, LNCS, Vol. 935, pp 278-297, ISBN: 3-540-60029-9, Torino, Italy, Springer-Verlag, London, Uk.

Li, J.Q. ; Fan, Y.S. \& Zhou, M.C. (2004). Performance Modeling and Analysis of Workflow. IEEE Transactions on Systems, Man, and Cybernetics - Part A: Systems and Humans, Vol. 34, No. 2, 2004, pp 229-242, ISSN: 1083-4427.

Lomazova, I. (2000). Nested Petri nets -a formalism for specification and verification of multi-agent distributed systems, Fundamenta informaticae, Vol 43, Issue 1-4, August 2000, pp. 195-214, ISSN:0169-2968, IOS Press Amsterdam, The Netherlands.

Lu, R. \& Sadiq, S. (2007). A Survey of Comparative Business Process Modeling Approaches. In Proceedings of $10^{\text {th }}$ International Conference on Business Information Systems BIS 2007, LNCS, pp. 82-94, ISBN: 978-3-540-72034-8, Poznan, Poland, Springer-Verlag.

Nwana, H. (1996). Software Agents: an Overview, Knowledge Engineering Review, Vol. 11, No 3, pp. 205-244.

Reijers, H.A. \& van der Aalst, W.M.P. (2005).The Effectiveness of Workflow Management Systems: Predictions and Lessons Learned. International Journal of Information Management, Vol. 25, No. 5, pp. 458-472. ISSN: 0268-4012.

Repetto, M.; Paolucci, M. \& Boccalatte, A. (2003). A design tool to Develop Agent-Based Workflow Management Systems, In Proc. Italian Workshop, from Objects to Agents: Intelligent Systems and Pervasive Computing (WOA2003), pp. 100-107, ISBN: 88-3711413-3, Villasimius, CA, Italy, September 2003. Pitagora Editrice Bologna.

Savarimuthu, B.T.R \& Purvis, M. (2004). A Collaborative Multi-Agent Based Workflow System, Knowledge-Based Intelligent Information and Engineering Systems KES'2004, Lecture Notes in Artificial Intelligence (LNAI), vol. 3214, pp. 1187-1193. ISSN: 0302-9743.

Savarimuthu, B.T.R.; Purvis, M. \& Fleurke, M. (2004). Monitoring and Controlling of a Multi-agent based Workflow System, In Proceedings of the second Workshop on Australasian Information Security, Data Mining and Web Intelligence, and Software Internationalisation, Vol. 32, pp. 127-132, Dunedin, New Zealand, 2004, Australian Computer Society, Inc. Darlinghurst, Australia. 
Shi, M.L.; Yang, G.; Xiang, Y. \& Wu, S. (1998). Workflow Management Systems: A Survey. In Proc. IEEE International Conference on Communication Technology, ICCT'98, pp. 1-5, Beijing, China, 1998.

Sibertin-Blanc, C. (1994). Cooperative Nets, In Proceedings of the 15th International Conference on Application and Theory of Petri Nets, LNCS, Vol. 815, pp. 471-490, ISBN:3-54058152-9, Zaragoza, Spain, June 1994, Springer-Verlag, London, UK.

Zisman, M.D. (1977). Representation, Specification and Automation of Office Procedures. PhD Thesis. Wharton School of Business, University of Pennsylvania. 1977.

Valk, R. (1998). Petri nets as token objects: An Introduction to Elementary Object Nets, In: Proceedings of the 19th Int. Conf. on Application and Theory of Petri Nets, LNCS, Vol. 1420, pp. 1-25, ISBN:3-540-64677-9, 1998, Springer-Verlag, London, UK.

Valk, R. (1991). Modeling Concurrency by Task/Flow EN Systems, In Proceedings of the $3^{\text {rd }}$ Workshop on Concurrency and Compositionality, pp. 207-215, GMD-Studien Nr. 191, Gesellschaft f. Mathematik und Datenverarbeitung, St. Augustin, Bonn.

van der Aalst, W.M.P. (1996). Three Good reasons for Using a Petri-net-based Workflow Management System. In Proceedings of the International Working Conference on Information and Process Integration in Enterprises (IPIC'96), pp. 179-201, Cambridge, Massachusetts, Nov. 1996.

van der Aalst, W.M.P. (1998). The Application of Petri Nets to Workflow Management. The Journal of Circuits, Systems and Computers, Vol. 8, No. 1, pp. 21-66.

van der Aalst, W.M.P. \& Anyanwu, K. (1999) Inheritance of Interorganizational Workflows to Enable Business-to-Business E-commerce. In Proceedings of the Second International Conference on Telecommunications and Electronic Commerce (ICTEC'99), pp. 141-157, October 1999, Nashville, Tennessee.

van der Aalst, W.M.P. \& Hee, K. (2002). Workflow Management: Models, Methods and Systems. MIT Press. ISBN: 0-262-72046-9, London.

van der Aalst, W.M.P. \& Hofstede, A. (2005). YAWL: Yet Another Workflow Language. Information Systems. Vol. 30 No. 4, pp.245-275. ISSN: 0306-4379.

Wang, M.; Wang, H. \& Xu, D. (2005), The design of intelligent workflow monitoring with agent technology, Knowledge-Based Systems, Vol. 18, Issue 6, pp. 257-266, ISBN: 0950-7051.

Weber, M. \& Kindler, E. (2003). The Petri Net Markup Language. In Petri Net Technology for Communication-Based Systems (LNCS), Volume 2472, Springer Berlin, pp 124-144. ISBN : 978-3-540-20538-8

WfMC. (1999). Workflow Management Coalition - Terminology \& Glossary. Technical report, The Workflow Management Coalition, Document Number WFMC-TC-1011, available at: http://www.wfmc.org/

Wooldridge, Michael (2001). Intelligent Agents: The Key Concepts, In Proceedings of the Multi-Agent-Systems and Applications II, (LNCS), Vol. 2322, pp 3-43, ISBN:3-54043377-5, Berling, Heidelberg, 2002, Springer-Verlag, London, UK.

Wooldridge Michael (2002), An Introduction to multiagent Systems, John Wiley \& Sons (Chichester, England), ISBN: 978-0-471-49691-5. 366 pp.

Yan, Y.; Maamar, Z. \& Shen, W. (2001). Integration of Workflow and Agent Technology for Business process Management, In Proceedings of the Sixth international Conference en CSCW in Design, pp. 420-426, ISBN: 0-660-18493-1, London, Ontario, Canada. July 2001. 


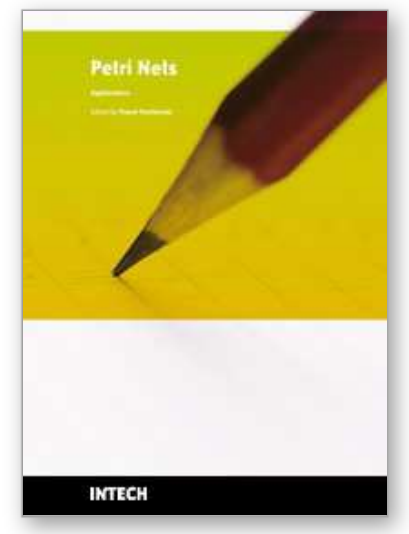

\author{
Petri Nets Applications \\ Edited by Pawel Pawlewski
}

ISBN 978-953-307-047-6

Hard cover, 752 pages

Publisher InTech

Published online 01, February, 2010

Published in print edition February, 2010

Petri Nets are graphical and mathematical tool used in many different science domains. Their characteristic features are the intuitive graphical modeling language and advanced formal analysis method. The concurrence of performed actions is the natural phenomenon due to which Petri Nets are perceived as mathematical tool for modeling concurrent systems. The nets whose model was extended with the time model can be applied in modeling real-time systems. Petri Nets were introduced in the doctoral dissertation by K.A. Petri, titled “"Kommunikation mit Automaten” and published in 1962 by University of Bonn. During more than 40 years of development of this theory, many different classes were formed and the scope of applications was extended. Depending on particular needs, the net definition was changed and adjusted to the considered problem. The unusual "flexibility" of this theory makes it possible to introduce all these modifications. Owing to varied currently known net classes, it is relatively easy to find a proper class for the specific application. The present monograph shows the whole spectrum of Petri Nets applications, from classic applications (to which the theory is specially dedicated) like computer science and control systems, through fault diagnosis, manufacturing, power systems, traffic systems, transport and down to Web applications. At the same time, the publication describes the diversity of investigations performed with use of Petri Nets in science centers all over the world.

\title{
How to reference
}

In order to correctly reference this scholarly work, feel free to copy and paste the following:

Marina Flores-Badillo and Ernesto Lopez-Mellado (2010). Multilevel Petri Nets for the Specification and Development of Workflow Automation Systems, Petri Nets Applications, Pawel Pawlewski (Ed.), ISBN: 978953-307-047-6, InTech, Available from: http://www.intechopen.com/books/petri-nets-applications/multilevelpetri-nets-for-the-specification-and-development-of-workflow-automation-systems

\section{INTECH}

open science | open minds

\section{InTech Europe}

University Campus STeP Ri

Slavka Krautzeka 83/A

51000 Rijeka, Croatia

Phone: +385 (51) 770447

Fax: +385 (51) 686166

www.intechopen.com

\section{InTech China}

Unit 405, Office Block, Hotel Equatorial Shanghai

No.65, Yan An Road (West), Shanghai, 200040, China 中国上海市延安西路65号上海国际贵都大饭店办公楼405单元

Phone: +86-21-62489820

Fax: +86-21-62489821 
(C) 2010 The Author(s). Licensee IntechOpen. This chapter is distributed under the terms of the Creative Commons Attribution-NonCommercial-ShareAlike-3.0 License, which permits use, distribution and reproduction for non-commercial purposes, provided the original is properly cited and derivative works building on this content are distributed under the same license. 